\title{
Value of Plethysmographic Pulse Amplitude Ratio With Valsalva Maneuver in Identifying Left Ventricular Dysfunction During COPD Exacerbation
}

\author{
Hamdi Boubaker MD, Mohamed Habib Grissa MD, Haithem Debbabi MD, Kaouthar Beltaief MD, \\ Zohra Dridi MD, Mohamed Fadhel Najjar MD, Wahid Bouida MD, Riadh Boukef MD, \\ Soudani Marghli MD, and Semir Nouira MD
}

\begin{abstract}
BACKGROUND: Left-ventricular dysfunction associated with COPD exacerbation is frequently underestimated. We tested whether finger plethysmography waveform change during the Valsalva maneuver could aid in the distinction between patients with COPD exacerbation with and without leftventricular dysfunction. METHODS: We included 102 subjects with COPD exacerbation and 51 subjects with acute heart failure admitted to the emergency department for acute dyspnea. Measurements of the plethysmographic pulse amplitude ratio under the Valsalva maneuver were obtained. The value of the plethysmographic pulse amplitude ratio in diagnosing left-ventricular dysfunction in COPD exacerbation subjects was quantified using the area under the receiver operating characteristic curves. Diagnostic performance of the plethysmographic pulse amplitude ratio was compared with the Boston score, and the net reclassification improvement was calculated to determine the incremental value in detecting left-ventricular dysfunction. RESULTS: Values of the plethysmographic pulse amplitude ratio were higher in COPD exacerbation subjects with left-ventricular dysfunction compared with those without left-ventricular dysfunction $(0.78$ vs $0.38, P=.01)$ but not significantly different from those of the acute heart failure group $(\mathbf{0 . 7 8})$. There was a significant correlation between plethysmographic pulse amplitude ratio values and brain natriuretic peptide serum levels. The area under the receiver operating characteristic curve for the plethysmographic pulse amplitude ratio to detect left-ventricular dysfunction among subjects with COPD exacerbation was significantly higher compared with that of the Boston score $(0.92$ vs $0.76, P=.02)$. Exposure to plethysmographic pulse amplitude ratio values increased the overall accuracy from Boston score-based predictions of left-ventricular dysfunction and provided a net reclassification improvement of 17.3\%. CONCLUSIONS: An increased plethysmographic pulse amplitude ratio associated with COPD exacerbation is a good predictor of left-ventricular dysfunction. This simple and noninvasive index could help emergency department physicians to detect or rule out leftventricular dysfunction in patients with COPD exacerbation who are able to perform the Valsalva maneuver. (ClinicalTrials.gov registration NCT01619540.) Key words: COPD; Valsalva maneuver; heart failure; left ventricular dysfunction; plethysmography. [Respir Care 2016;61(4):439-446. (C) 2016 Daedalus Enterprises]
\end{abstract}

\section{Introduction}

COPD is a frequent cause of emergency department visits and hospital admission for exacerbation of symp-

Drs Boubaker, Grissa, Debbabi, Beltaief, Bouida, and Nouira are affiliated with the Emergency Department, Fattouma Bourguiba University Hospital Monastir, Tunisia and the Research Laboratory (LR12SP18), University of Monastir, Monastir, Tunisia. Dr Dridi is affiliated with the Cardiology Department, Fattouma Bourguiba University Hospital, Monastir, Tunisia. Dr Najjar is affiliated with the Biochemistry Department, Fattouma Bourguiba University Hospital, Monastir, Tunisia. Dr Boukef is affiliated with the Emergency Department, Sahloul University Hospital, Sousse, Tunisia and the Research Laboratory (LR12SP18), University of Monastir, Monastir, toms. ${ }^{1}$ The most common cause of COPD exacerbation is infection of the tracheobronchial tree. ${ }^{2}$ The role of congestive heart failure is usually underestimated and pre-

\footnotetext{
Tunisia. Dr Marghli is affiliated with the Emergency Department, Tahar Sfar University Hospital, Mahdia, Tunisia and the Research Laboratory (LR12SP18), University of Monastir, Monastir, Tunisia.

The authors have disclosed no conflicts of interest.

Correspondence: Semir Nouira MD, Emergency Department, Fattouma Bourguiba University Hospital, 5000 Monastir, Tunisia. E-mail: Semir.nouira@rns.tn.
}

DOI: $10.4187 /$ respcare.04091 
cludes early institution of appropriate medical therapy. Distinguishing cardiac decompensation of COPD exacerbation at the time of emergency department presentation is often challenging because there is a great overlap between symptoms of cardiac and respiratory illness. ${ }^{3,4}$ Echocardiography is the standard method for detecting left ventricular dysfunction, but it is not usually available in the emergency department and requires great skill and knowledge by the operator. Although natriuretic peptides have been used increasingly in this situation, their use could be limited because they can be increased in many other conditions. ${ }^{5,6}$ Diagnostic uncertainty using brain natriuretic peptide (BNP) is likely to be higher in patients with COPD exacerbation because both left and right ventricular dysfunction with pulmonary hypertension could influence BNP level. Accordingly, additional strategies are needed to improve the diagnostic accuracy of left-ventricular dysfunction in COPD exacerbation. Several reports have demonstrated that blood pressure response to the Valsalva maneuver could predict the presence of left-ventricular dysfunction in undifferentiated patients presenting with dyspnea. ${ }^{7-10}$ However, the diagnostic value of this test in patients with COPD exacerbation is unknown. Recently, it was shown that finger photoplethysmography during the Valsalva maneuver could reflect left ventricular filling, which could greatly simplify the diagnostic approach of left-ventricular dysfunction in subjects with acute dyspnea in the emergency department setting. ${ }^{11}$ The goal of the present study was to evaluate the value of the Valsalva maneuver test using photoplethysmography in COPD exacerbation subjects for the diagnosis of left-ventricular dysfunction.

\section{Methods}

\section{Study Design and Setting}

This cross-sectional study was performed in the emergency department of Fattouma Bourguiba University Hospital (Monastir, Tunisia) between May 2011 and June 2013. The study was approved by the Ethical Committee of our institution. Patients diagnosed with COPD exacerbation were screened for inclusion except during the night shift and the weekend. Exacerbation of COPD was defined as an increase in cough and dyspnea and a change in sputum volume and purulence. The definition of COPD was based on pulmonary function tests when available, clinical history, physical examination, arterial blood gas, and chest radiograph findings. Exclusion criteria were patients $<18 \mathrm{y}$ old, severe renal or coronary disease, and evident causes of dyspnea. Patients with hemodynamic instability or who were deemed unable to perform the Valsalva maneuver were also excluded, as were those with clinically diagnosed mitral or aortic valvular stenosis and constrictive

\section{QUICK LOOK}

\section{Current knowledge}

Detection of left ventricular dysfunction in acute COPD exacerbation is challenging in clinical practice because there is a great overlap between symptoms of cardiac and respiratory illness. This often leads to inappropriate therapeutic intervention and increased morbidity and mortality. There is no study that has investigated the test characteristics of finger photoplethysmography changes under the Valsalva maneuver in such patients.

\section{What this paper contributes to our knowledge}

A bedside Valsalva maneuver coupled to finger photoplethysmography changes to measure the plethysmographic pulse amplitude ratio could help to detect or rule out left-ventricular dysfunction. A plethysmographic pulse amplitude ratio of $>0.5$ was a good predictor of left-ventricular dysfunction in COPD exacerbation.

pericarditis. A convenience sample of subjects admitted to the emergency department with a standard diagnosis of acute heart failure was enrolled in the study as a control group. Written informed consent was obtained from all subjects.

\section{Study Protocol}

After enrollment, subject's demographic and clinical characteristics with routine blood test results were recorded. Treating physicians were asked to complete a Boston score form for congestive heart failure. ${ }^{12}$ All included subjects underwent BNP measurement and echocardiography at admission within $60 \mathrm{~min}$ of inclusion in the study. Echocardiography was performed by one of the 4 full-time senior emergency physicians using a portable ultrasound system (SonoSite, Bothell, Washington) with a $2.5-\mathrm{MHz}$ array probe. The echocardiographic examination included Mmode and 2-dimensional examination and pulsed Doppler analysis of mitral inflow. A calibrated Valsalva maneuver lasting $10 \mathrm{~s}$ with an expiratory effort pressure of $25 \mathrm{~cm} \mathrm{H}_{2} \mathrm{O}$ was performed in all included subjects. A disposable mouthpiece and a disposable pressure transducer (type TNF-R, Vigo-Spectramed, Bilthoven, Netherlands) were connected by a homemade stainless steel tube. Before the measurement, the Valsalva maneuver was practiced to ensure that the subject was able to perform the maneuver. In all included subjects, the plethysmographic pulse amplitude ratio was measured by analyzing Valsalva maneuverinduced changes in the amplitude of the plethysmo- 


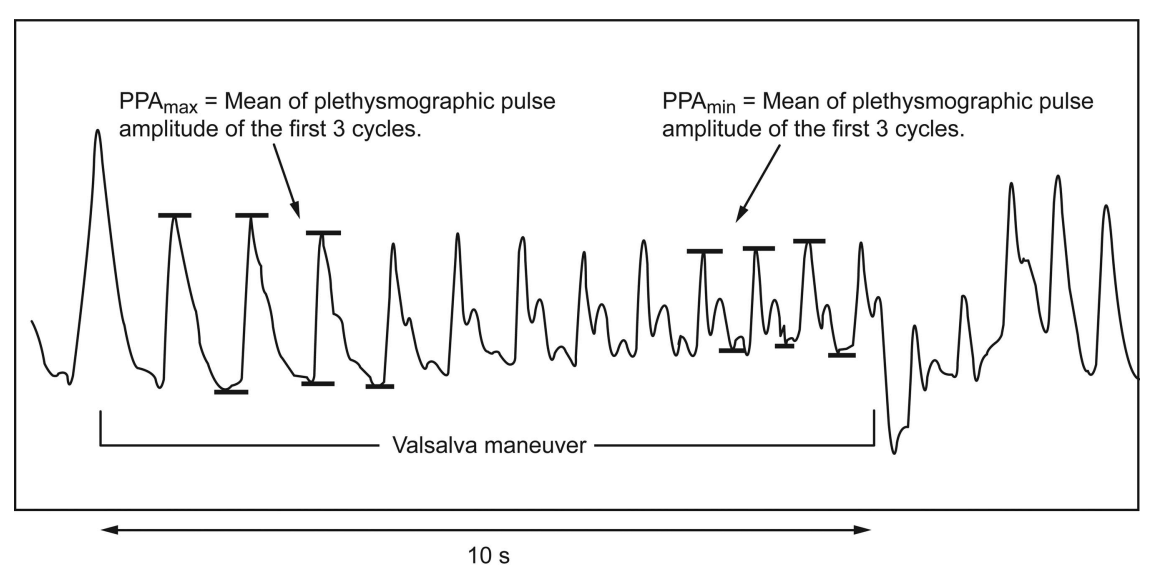

Fig. 1. Pulse amplitude ratio using plethysmographic waveform recording under the Valsalva maneuver in a subject with COPD exacerbation associated with ventricular dysfunction. Plethysmographic pulse amplitude ratio = minimum plethysmographic pulse amplitude $\left(\mathrm{PPA}_{\min }\right) /$ maximum plethysmographic pulse amplitude $\left(\mathrm{PPA}_{\max }\right)$. In the example of this figure, the plethysmographic pulse amplitude ratio $=0.60$.

graphic pulse signals using an analog numeric system (Biopac Systems, Goleta, California). The plethysmographic pulse amplitude ratio was calculated as the ratio of the mean of the last 3 successive plethysmographic pulse signals under the Valsalva maneuver and the mean of the first 3 successive photoplethysmographic pulse signals obtained in phase 1 of the Valsalva maneuver (Fig. 1). All studies were performed with subjects in a semi-recumbent position with the head at a $30^{\circ}$ position. For each subject, measurements were repeated if the subject could tolerate the maneuver. A third measurement was required if the difference between the first 2 values was $>10 \%$. When $>1$ measurement of the plethysmographic pulse amplitude ratio was obtained, the average value was recorded. The physician performing the Valsalva maneuver test was unaware of the subject's final diagnosis. Two independent reviewers (one emergency physician and one cardiologist), blinded to the Valsalva maneuver test result, established the diagnosis of left-ventricular dysfunction on the basis of clinical data, BNP levels, and echocardiographic findings.

The inter-observer variability of plethysmographic pulse amplitude ratio measurements was determined in a blinded fashion. It was assessed in 50 randomly selected sets of measurements by calculating the ratio (expressed as a percentage) of the difference between the values obtained by each observer (expressed as absolute value) divided by the mean of the 2 values.

\section{Statistical Analysis}

Subjects with COPD exacerbation were divided into 2 groups: subjects with left-ventricular dysfunction (COPD exacerbation with left-ventricular dysfunction group) and subjects without left-ventricular dysfunction (COPD exac- erbation without left-ventricular dysfunction group). Subjects with acute heart failure were designated as a control group. Variables are expressed as mean $\pm \mathrm{SD}$, median (interquartile range), or value (95\% CI), as appropriate. Comparison between the 3 groups was examined by using analysis of variance for normally distributed numerical variables and the Kruskal-Wallis test for non-normally distributed numerical variables. Categorical variables were presented as a proportion and compared with a chi-square test between the 3 groups. Spearman rank correlation was performed to evaluate the relationship of plethysmographic pulse amplitude ratio values and BNP levels. Receiver operating characteristic curves were constructed, and area under the curve was measured for the plethysmographic pulse amplitude ratio and Boston score for predicting leftventricular dysfunction.

We estimated a sample size of 110 subjects with COPD exacerbation, assuming an anticipated plethysmographic pulse amplitude ratio sensitivity of at least $90 \%$ with a power $(1-\beta)=80 \%$, and a probability of type- 1 error $(\alpha)=0.05$. Sensitivity and specificity, positive and negative predictive values, accuracy, and likelihood ratios of positive and negative results (with 95\% confidence intervals) were calculated using the best cut-off value of the plethysmographic pulse amplitude ratio and Boston score. To assess the incremental value of the plethysmographic pulse amplitude ratio for the diagnosis of left-ventricular dysfunction beyond the Boston score, reclassification analysis was performed, utilizing the method of net reclassification improvement. ${ }^{13} \mathrm{~A}$ net reclassification improvement significantly $>0$ indicates incremental diagnostic benefit. All tests were 2 -tailed, and a $P$ value of $<.05$ was considered statistically significant. Calculations were performed with SPSS 13 for Windows (SPSS, Chicago, Illinois). 


\section{Left Ventricular Dysfunction in COPD Exacerbation}

Table 1. Subjects' Characteristics at Admission

\begin{tabular}{|c|c|c|c|}
\hline \multirow{2}{*}{ Characteristics } & \multirow{2}{*}{ Acute Heart Failure $(n=51)$} & \multicolumn{2}{|c|}{ COPD Exacerbation } \\
\hline & & With LVD $(n=49)$ & Without LVD $(n=53)$ \\
\hline Age, mean \pm SD y & $70 \pm 9$ & $71 \pm 10^{*}$ & $67 \pm 10$ \\
\hline Male/female sex, $n$ & $32 / 19$ & $39 / 10$ & $40 / 13$ \\
\hline \multicolumn{4}{|l|}{ Previous medical history, $n(\%)$} \\
\hline Arterial hypertension & $18(35)$ & $25(51)$ & $13(25)$ \\
\hline Diabetes & $10(20)$ & $16(33)$ & $15(28)$ \\
\hline Coronary artery disease & $16(31)$ & $7(14)^{*}$ & $1(2)$ \\
\hline Systolic BP, mean \pm SD mm Hg & $140 \pm 26$ & $136 \pm 27$ & $131 \pm 25$ \\
\hline Diastolic $\mathrm{BP}$, mean $\pm \mathrm{SD} \mathrm{mm} \mathrm{Hg}$ & $71 \pm 18$ & $78 \pm 17$ & $75 \pm 18$ \\
\hline Heart rate, mean $\pm \mathrm{SD}$ beats/min & $101 \pm 20$ & $97 \pm 19$ & $103 \pm 21$ \\
\hline $\mathrm{P}_{\mathrm{aO}_{2}}$, mean $\pm \mathrm{SD} \mathrm{mm} \mathrm{Hg}$ & $66 \pm 17$ & $59 \pm 14$ & $68 \pm 23$ \\
\hline $\mathrm{P}_{\mathrm{aCO}_{2}}$, mean $\pm \mathrm{SD} \mathrm{mm} \mathrm{Hg}$ & $34 \pm 4$ & $57 \pm 6^{*}$ & $51 \pm 4$ \\
\hline BNP serum level, median (IQR) $\mathrm{pg} / \mathrm{mL}$ & $871(620-1258)$ & $450(405-740)^{*}$ & $68(28-150)$ \\
\hline $\mathrm{LVEF}$, mean $\pm \mathrm{SD} \%$ & $41 \pm 7 \dagger$ & $44 \pm 5^{*}$ & $63 \pm 6$ \\
\hline E/A ratio & 1.06 & 0.97 & 1.10 \\
\hline $\begin{array}{l}\text { *P } P<.05 \text { COPD exacerbation with LVD vs without } \\
\dagger P<.05 \text {, acute heart failure group vs COPD exacert } \\
\text { LVD = left-ventricular dysfunction } \\
\text { BP = blood pressure } \\
\text { BNP = brain natriuretic peptide } \\
\text { IQR = interquartile range } \\
\text { LEVF = left-ventricular ejection fraction } \\
\text { E/A ratio = early/late diastolic transmitral velocity ra }\end{array}$ & LVD group. & & \\
\hline
\end{tabular}

\section{Results}

\section{Characteristics of the Patients}

Baseline clinical and demographic characteristics of the study groups are presented in Table 1 . One hundred ninetythree patients were screened for the protocol, which was completed in 102 subjects with COPD exacerbation and 51 subjects of the acute heart failure group. The protocol was not completed in 40 patients because of failure to obtain a good plethysmographic signal $(n=21)$ or sustained ventricular arrhythmia $(n=8)$. Inability to perform the Valsalva maneuver was observed in 11 subjects (5.6\%). The diagnosis of left-ventricular dysfunction was established in 49 subjects of the COPD exacerbation group. The inter-expert agreement on the presence of left-ventricular dysfunction was good (kappa $=0.77)$. The prevalence of hypertension and diabetes was similar in all groups. Subjects in the COPD exacerbation with left-ventricular dysfunction group were older, more frequently had a history of coronary artery disease, and were more hypercapnic than those in the group of COPD exacerbation without left-ventricular dysfunction. There was no significant difference between the acute heart failure and COPD exacerbation with left-ventricular dysfunction groups regarding BNP values at emergency department admission. In subjects with COPD exacerbation, median BNP values were significantly higher in those with left-ventricular dys- function compared with those without left-ventricular dysfunction. Echocardiographic results showed that subjects in the acute heart failure group and those in the COPD exacerbation with left-ventricular dysfunction group had a significantly lower left ventricular ejection fraction compared with the COPD exacerbation group without leftventricular dysfunction. Diastolic mitral flow Doppler parameters were not significantly different between the 3 groups.

\section{Plethysmographic Pulse Amplitude Ratio}

The mean duration between emergency department admission and the Valsalva maneuver test was $178 \pm 85 \mathrm{~min}$. For plethysmographic pulse amplitude ratio measurements, the inter-observer variability was $7.0 \pm 4.1 \%$, and the intra-observer variability was $4.4 \pm 2.5 \%$. Median plethysmographic pulse amplitude ratio values were similar between the COPD exacerbation with left-ventricular dysfunction group and the acute heart failure group (0.78 [interquartile range $0.71-0.84]$ vs 0.78 [0.60-0.86], respectively). Compared with these 2 groups, median plethysmographic pulse amplitude ratio values in the COPD exacerbation without left-ventricular dysfunction group were significantly lower $(0.38$ [interquartile range 0.23 0.52 ], $P=.01$ ) (Fig. 2). We found a significant correlation between plethysmographic pulse amplitude ratio values and BNP serum levels (Fig. 3). Using multiple logistical 


\section{Left Ventricular Dysfunction in COPD Exacerbation}

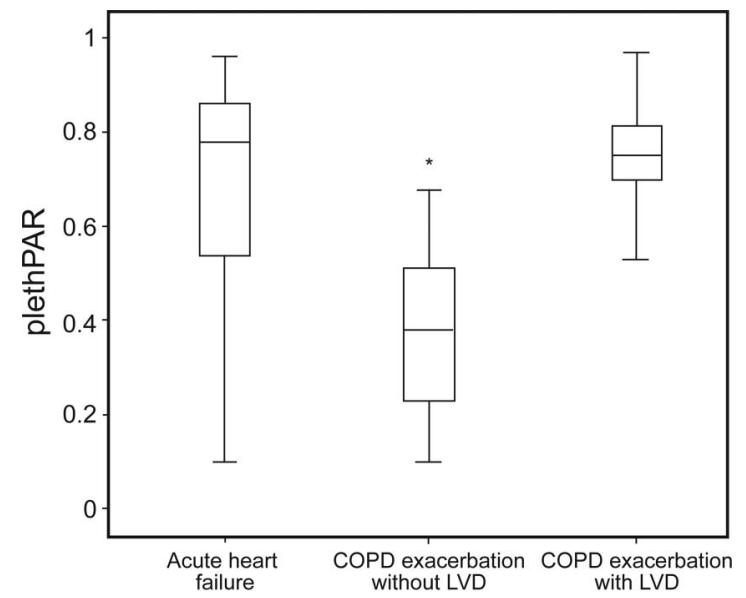

Fig. 2. Box plots showing plethysmographic pulse amplitude ratio (plethPAR) in the 3 study groups: acute heart failure group, subjects with COPD exacerbation and left ventricular dysfunction (COPD exacerbation with LVD), and subjects with COPD exacerbation without left ventricular dysfunction (COPD exacerbation without LVD). The line in each box indicates the median. The upper and lower limits of each box indicate the 75th and 25th percentiles, respectively. Whiskers denote the 90th and 10th percentiles. ${ }^{*}, P<.05$ vs acute heart failure and COPD exacerbation with leftventricular dysfunction groups.

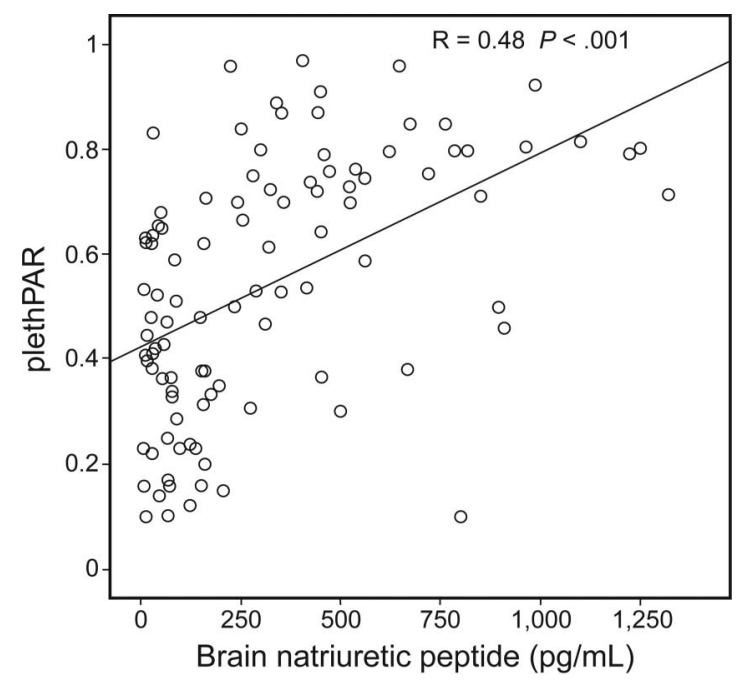

Fig. 3. Correlation between plethysmographic pulse amplitude ratio (plethPAR) and brain natriuretic peptide in subjects with COPD exacerbation. The regression line and correlation coefficient are shown.

regression including only COPD subjects with input of significant univariate variables, we found that previous history of arterial hypertension was the most independently predictive variable of left-ventricular dysfunction (Table 2). The performance of plethysmographic pulse amplitude ratio in predicting left ventricular dysfunction at a cutoff value of 0.5 is summarized in Table 3 .
Table 2. Variables Independently Predictive of Left-Ventricular Dysfunction in Subjects With COPD Exacerbation

\begin{tabular}{lcc}
\hline \hline \multicolumn{1}{c}{ Variables } & Odds Ratio & 95\% CI \\
\hline Previous history of arterial hypertension & 3.20 & $1.38-742$ \\
Serum glucose level & 1.38 & $1.10-1.72$ \\
Plethysmographic pulse amplitude ratio & 1.15 & $1.08-1.22$ \\
\hline
\end{tabular}

Table 3. Performance of Plethysmographic Pulse Amplitude Ratio in Predicting Left-Ventricular Dysfunction at a Cutoff Value of 0.5

\begin{tabular}{lcc}
\hline \hline \multicolumn{1}{c}{ Parameters } & Values & $95 \%$ CI \\
\hline Sensitivity, \% & 90 & $78-96$ \\
Specificity, \% & 75 & $62-85$ \\
Positive predictive value, \% & 77 & $66-88$ \\
Negative predictive value, \% & 89 & $80-98$ \\
Positive likelihood ratio & 3.66 & $2.26-5.93$ \\
Negative likelihood ratio & 0.14 & $0.06-0.32$ \\
\hline
\end{tabular}

\section{Comparison Between Plethysmographic Pulse Amplitude Ratio and Boston Score}

The mean Boston score was $9.7 \pm 1.9$ in the acute heart failure group and $8.3 \pm 2.0$ in subjects with COPD exacerbation and left-ventricular dysfunction $(P=.23)$. Mean Boston score was significantly higher in these 2 groups compared with the COPD exacerbation without left-ventricular dysfunction group $(6.1 \pm 2.5, P<.05)$. The area under the receiver operating characteristic curve using the plethysmographic pulse amplitude ratio to detect left-ventricular dysfunction among subjects with COPD exacerbation was 0.92 (95\% CI 0.87-0.97) The area under the receiver operating characteristic curve for Boston score was 0.76 (95\% CI 0.66-0.85); it was significantly lower compared with the plethysmographic pulse amplitude ratio (Fig. 4). Exposure to plethysmographic pulse amplitude ratio values improved the overall accuracy of Boston scorebased predictions of left-ventricular dysfunction (from $71.5 \%$ before to $82 \%$ after the plethysmographic pulse amplitude ratio test, $P=.03$ ). Left ventricular dysfunction was reclassified after plethysmographic pulse amplitude ratio value exposure in 19 subjects. Net reclassification improvement after exposure to plethysmographic pulse amplitude ratio was $17.3 \%$ (95\% CI 14.2-20.4). This value is significantly different from zero, indicating an incremental benefit from plethysmographic pulse amplitude ratio testing beyond the Boston score alone.

\section{Discussion}

In the present study, we demonstrated the following: (1) left ventricular dysfunction in COPD exacerbation is as- 


\section{Left Ventricular Dysfunction in COPD Exacerbation}

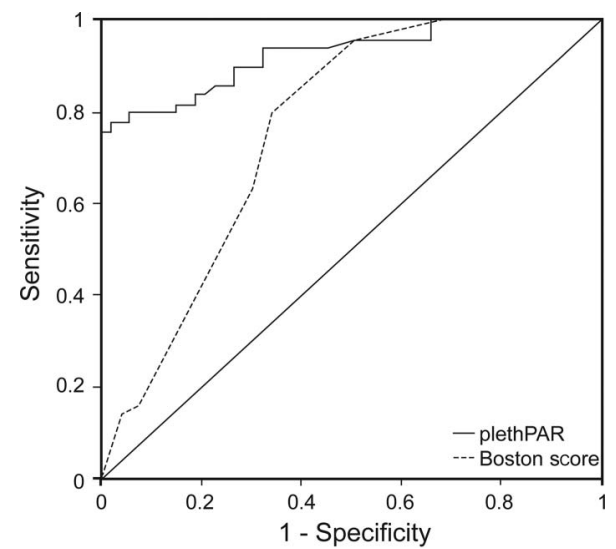

Fig. 4. Receiver operating characteristic curves for the diagnosis of left ventricular dysfunction related to plethysmographic pulse amplitude ratio (plethPAR) and to Boston score. Area under the curve was 0.92 (95\% Cl 0.87-0.97) and 0.76 (95 Cl 0.66-0.85), respectively, for the plethysmographic pulse amplitude ratio and Boston score.

sociated with an increased plethysmographic pulse amplitude ratio; (2) the plethysmographic pulse amplitude ratio is well correlated with serum levels of BNP; (3) a plethysmographic pulse amplitude ratio value of $>0.5$ is a good predictor of left-ventricular dysfunction in COPD exacerbation; and (4) the plethysmographic pulse amplitude ratio had the potential to improve diagnostic accuracy of leftventricular dysfunction beyond a clinical model, such as the Boston score.

Early diagnosis of the presence of left-ventricular dysfunction is a significant clinical challenge in patients admitted to the emergency department with COPD exacerbation. This is an important issue because identifying heart failure in COPD exacerbation can immediately translate to specific treatment of heart failure. ${ }^{14}$ The accepted standard methods to assess cardiac function, including invasive cardiac catheterization and echocardiography, are not always available in most emergency departments, and they often require a lengthy training period. The potential usefulness of pulse amplitude ratio measurement using the Valsalva maneuver as a diagnostic test of left ventricular dysfunction in patients with acute dyspnea was previously suggested. ${ }^{8,9,10,15}$ Moreover, noninvasive recording systems that provide reproducible estimates of arterial response to the Valsalva maneuver showed that these methods can accurately predict pulmonary capillary wedge pressure and its changes in response to medical therapy. ${ }^{8}$ This is very important because photoplethysmography is routinely used and widely available in the emergency department. Recently, Silber et al ${ }^{11}$ demonstrated that the pulse amplitude ratio measured by photoplethysmography was well correlated with left ventricular end-diastolic pressure. Our study is the first to evaluate this noninvasive test in a selected group of subjects with COPD exacerbation. This raises the question of whether COPD-related right ventricular dysfunction could affect the relationship between altered left ventricular function and response to the Valsalva maneuver. ${ }^{16}$ Indeed, the pulse amplitude ratio response to the Valsalva maneuver might reflect left ventricular function, ${ }^{17}$ right-sided pressure, ${ }^{18}$ or both. ${ }^{19,20}$ Although we did not assess right ventricular function in our subjects, our results indicated that left ventricular function is probably the major determinant of pulse amplitude ratio values even in the presence of right ventricular dysfunction. ${ }^{9,20}$ Among the mechanisms proposed to explain the abnormal response to the Valsalva maneuver in patients with left-ventricular dysfunction, the most likely is the shift of blood from the congested pulmonary vascular bed to the left atrium. ${ }^{21}$ Pulmonary blood volume serves as a tank that maintains left ventricular filling despite the decrease in systemic venous return during the Valsalva maneuver. In addition, the decrease in right ventricular volume during the strain phase would limit ventricular interdependence and limit the potential effect of right ventricular enlargement on left ventricular filling in patients with COPD exacerbation. Of note, correlation between pulse amplitude ratio and BNP in our study is consistent with the value of this index as a left-ventricular dysfunction marker. The close correlation between pulse amplitude ratio and BNP was previously demonstrated by Brunner-La Rocca et $\mathrm{a}^{22}$ in subjects with stable congestive heart failure. Moreover, using multiple logistic regression, we found that the plethysmographic pulse amplitude ratio is an independent predictive variable of left-ventricular dysfunction. Specifically, a plethysmographic pulse amplitude ratio value of $>0.5$ could be considered as a "rule-in" cut point for the presence of leftventricular dysfunction with a sensitivity of $90 \%$ and a specificity of $75 \%$. In a previous study conducted in 42 stable subjects undergoing right heart catheterization, Weilenmann et $\mathrm{al}^{23}$ found that a pulse amplitude ratio cutoff of $>0.7$ predicted the presence of an elevated pulmonary capillary wedge pressure (ie, $>15 \mathrm{~mm} \mathrm{Hg}$ ) with a sensitivity of $91 \%$, a specificity of $95 \%$, and a diagnostic accuracy of $93 \%$. Using a finger photoplethysmograph, as in our study, Silber et $\mathrm{al}^{11}$ found that the best pulse amplitude ratio cutoff is 0.4 , which is moderately different from our findings. Threshold differences between studies are likely to be explained by differences in subject selection, methods of pulse amplitude ratio measurements, or criteria used to define left-ventricular dysfunction. Of note, the Boston score was not found to be an independent predictor of left-ventricular dysfunction with an area under the receiver operating characteristic curve lower than that of the pulse amplitude ratio index. However, our study showed that combination of these 2 parameters had a significantly better diagnostic accuracy than each index alone. The yield of adding routine pulse amplitude ratio testing to the clinical diagnostic approach using the Boston score is 


\section{Left Ventricular Dysfunction in COPD Exacerbation}

approximately $20 \%$. Such an early and simple method of left-ventricular dysfunction diagnosis in COPD exacerbation will probably help the emergency physician in everyday clinical practice.

Potential limitations of our study data merit consideration. First, the study population may not fully represent all patients with COPD exacerbation, in particular those who were in a severe condition and unable to perform the Valsalva maneuver. To limit the proportion of these subjects, we chose Valsalva maneuver parameters (expiratory effort pressure of $25 \mathrm{~cm} \mathrm{H}_{2} \mathrm{O}$ and expiratory effort duration of $10 \mathrm{~s}$ ) that most patients with COPD exacerbation would be likely to achieve. Indeed, in our study, the proportion of these subjects was low (5.6\%) and unlikely to alter significantly the validity of our results. In patients unable to perform the Valsalva maneuver, alternative investigations, such as echocardiography, are needed. Second, we acknowledge that left-ventricular dysfunction misclassification bias is possible, especially with respect to the fact that BNP cutoff values for left-ventricular dysfunction diagnosis are not well defined in patients with COPD exacerbation. However, we believe this bias would be reduced by the fact that in addition to BNP values, we required systematic echocardiographic criteria and the agreement of 2 clinical experts to define left-ventricular dysfunction. Third, the sensitivity and specificity reported here are based on a threshold of the pulse amplitude ratio derived from the same sample subjects' data. A prospective study on a different population is required for a formal assessment of pulse amplitude ratio performance. Last, this study did not examine the ability of Valsalva maneuver testing to affect treatment decisions or outcomes. Indeed, many patients with COPD exacerbation will have some degree of cardiac dysfunction, but the presence of left-ventricular dysfunction does not necessarily guide care, especially if left-ventricular dysfunction is baseline for the patient. Further studies are needed to demonstrate that early determination of left-ventricular dysfunction in the course of COPD exacerbation, whatever the method used, could potentially lead to a specific treatment or avoidance of an unnecessary one. Nevertheless, the Valsalva maneuver using plethysmography is a simple diagnostic test that takes only a few minutes to perform and represents a significant advantage over serum biomarker strategies or echocardiography.

\section{Conclusion}

A significant proportion of subjects with COPD exacerbation had associated left-ventricular dysfunction. Our study showed that a bedside Valsalva maneuver coupled with finger photoplethysmography could help to detect or rule out left-ventricular dysfunction in these patients, which may have a significant impact on their clinical outcome.

\section{ACKNOWLEDGMENTS}

We thank Dr Asma Belghith for helpful revision of the statistical analysis of the study.

\section{REFERENCES}

1. Mannino DM. Epidemiology and global impact of chronic obstructive pulmonary disease. Semin Respir Crit Care Med 2005;26(2): 204-210.

2. White AJ, Gompertz S, Stockley RA. Chronic obstructive pulmonary disease. 6: The aetiology of exacerbations of chronic obstructive pulmonary disease. Thorax 2003;58(1):73-80.

3. Abroug F, Ouanes-Besbes L, Nciri N, Sellami N, Addad F, Hamda $\mathrm{KB}$, et al. Association of left-heart dysfunction with severe exacerbation of chronic obstructive pulmonary disease: diagnostic performance of cardiac biomarkers. Am J Respir Crit Care Med 2006; 174(9):990-996.

4. McCullough PA, Hollander JE, Nowak RM, Storrow AB, Duc P, Omland $\mathrm{T}$, et al. Uncovering heart failure in patients with a history of pulmonary disease: rationale for the early use of B-type natriuretic peptide in the emergency department. Acad Emerg Med 2003;10(3): 198-204.

5. Maisel A, Mueller C, Adams K Jr., Anker SD, Aspromonte N, Cleland JG, et al. State of the art: using natriuretic peptide levels in clinical practice. Eur J Heart Fail 2008;10(9):824-839.

6. McCullough PA, Nowak RM, McCord J, Hollander JE, Herrmann $\mathrm{HC}$, Steg PG, et al. B-type natriuretic peptide and clinical judgment in emergency diagnosis of heart failure: analysis from breathing not properly (BNP) multi study. Circulation 2002;106(4):416-422.

7. Zema MJ, Masters AP, Margouleff D. Dyspnea: the heart or the lungs? Diffrentiation at bedside by use of the simple Valsalva maneuver. Chest 1984;85(1):59-64.

8. McIntyre KM, Vita JA, Lambrew CT, Freeman J, Loscalzo J. A. Noninvasive method of predicting pulmonary-capillary wedge pressure. N Engl J Med 1992;327(24):1715-1720.

9. Schmidt DE, Shah PK. Accurate detection of elevated left ventricular filing pressure by a simplified bedside application of the Valsalva maneuver. Am J Cardiol 1993;71(5):462-465.

10. Sharma GV, Woods PA, Lambrew CT, Berg CM, Pietro DA, Rocco $\mathrm{TP}$, et al. Evaluation of a noninvasive system for determining left ventricular filling pressure. Arch Intern Med 2002;162(18):20842088.

11. Silber HA, Trost JC, Johnston PV, Maughan WL, Wang NY, Kasper EK, et al. Finger photoplethysmography during the valsava maneuver reflects left ventricular filling pressure. Am J Physiol Heart Circ Physiol 2012;302(10):H2043-H2047.

12. Nazerian P, Vanni S, Zanobetti M, Polidori G, Pepe G, Federico R, et al. Diagnostic accuracy of emergency Doppler echocardiography for identification of acute left ventricular heart failure in patients with acute dyspnea: comparison with Boston criteria and N-terminal prohormone brain natriuretic peptide. Acad Emerg Med 2010;17(1): 18-26.

13. Pencina MJ, D'Agostino RB Sr, D’Agostino RB Jr., Vasan RS. Evaluating the added predictive ability of a new marker: from area under the ROC curve to reclassification and beyond. Stat Med 2008; 27(2):157-172; discussion 207-212.

14. Mentz RJ, Fiuzat M, Kraft M, Lindenfeld J, O'Connor CM. Bronchodilators in heart failure patients with COPD: is it time for a clinical trial? J Card Fail 2012;18(5):413-422.

15. Zema MJ, Caccavano M, Kligfield P. Detection of left ventricular dysfunction in ambulatory subjects with the bedside Valsalva maneuver. Am J Med 1983;75(2):241-248. 


\section{Left Ventricular Dysfunction in COPD Exacerbation}

16. Hébert JL, Coirault C, Zamani K, Fontaine G, Lecarpentier Y, Chemla D. Pulse pressure response to the strain of the valsava maneuver in humans with preserved systolic function. J Appl Physiol 1998;85(3): 817-823.

17. Braunwald E, Colucci WS, Grossman W. Clinical aspects of heart failure: high output heart failure; pulmonary edema. In: Braunwald E, editor. Heart disease: a textbook of cardiovascular medicine. Philadelphia, PA: Saunders; 1997:445-470.

18. Judson, WE, Hatcher JD, Wilkins RW. Blood pressure responses to the Valsalva maneuver in cardiac patients with and without congestive failure. Circulation 1955;11(6):889-899.

19. Bernardi L, Saviolo R, Spodick DH. Do hemodynamic responses to the Valsalva maneuver reflect myocardial dysfunction? Chest 1989; 95(5):986-991.
20. Opotowsky AR, Ojeda J, Rogers F, Arkles J, Liu T, Forfia PR. Blood pressure response to the Valsalva maneuver: a simple bedside test to determine the hemodynamic basis of pulmonary hypertension. J Am Coll Cardiol 2010;56(16):1352-1353.

21. Zema MJ, Restivo B, Sos T, Sniderman KW, Kline S. Left ventricular dysfunction-bedside Valsalva manoeuvre. Br Heart J 1980;44(5):560-569.

22. Brunner-La Rocca HP, Weilenmann D, Rickli H, Follath F, Kiowski W. Is blood pressure response to the Valsalva maneuver related to neurohormones, exercise capacity, and clinical findings in heart failure? Chest 1999;116(4):861-867.

23. Weilenmann D, Rickli H, Follath F, Kiowski W, Brunner-La Rocca HP. Noninvasive evaluation of pulmonary capillary wedge pressure by $\mathrm{BP}$ response to the vaslsalva maneuver. Chest 2002;122(1):140145 . 